\title{
Seasonal fluctuation in vitamin $D$ status is associated with indices of bone health in UK South Asian but not Caucasian women living at $51^{\circ} \mathrm{N}$
}

\author{
A. L. Darling ${ }^{1}$, J. L. Berry ${ }^{2}$, F. Gossiel ${ }^{3}$, R. Hannon ${ }^{3}$, R. Eastell ${ }^{3}$ and S. A. Lanham-New ${ }^{1}$ \\ ${ }^{1}$ Faculty of Health and Medical Sciences, University of Surrey, GU2 7XH, UK, ${ }^{2}$ Vitamin D Research Group, University of \\ Manchester, M13 9WL, UK and ${ }^{3}$ Department of Human Metabolism, University of Sheffield, S10 2RX, UK
}

It has been suggested that findings of increased risk of some cancers in countries with high vitamin D status $(25(\mathrm{OH}) \mathrm{D}$; 25-dihydroxyvitamin D) could be due to slow adaption of the vitamin D hydroxylase enzymes (CYP27B1 and CYP24) to fluctuating $25(\mathrm{OH}) \mathrm{D}^{(1)}$. This begs the question as to whether seasonal fluctuation or 'cycling' of $25(\mathrm{OH}) \mathrm{D}$ could be detrimental to bone. Indeed, a recent review discussed a direct correlation between $25(\mathrm{OH}) \mathrm{D}$ and bone indices ${ }^{(2)}$.

The present investigation aimed to assess whether seasonal fluctuation of $25(\mathrm{OH}) \mathrm{D}$ is associated with bone health. A subset of 248 women (South Asian ( $n$ 55) and Caucasian ( $n$ 193)) who took part in the 2006-2007 D-FINES (Vitamin D, Food Intake, Nutrition and Exposure to Sunlight in Southern England) study was analysed. During D-FINES, subjects had blood taken in four seasons for determination of $\overline{25}(\mathrm{OH}) \mathrm{D}$ and in autumn and spring they had a dual X-ray absorptiometry (DXA) scan (Hologic). In a further sub-group of 65 women (South Asian ( $n$ 30) and Caucasian ( $n$ 35)) blood samples were assessed for the bone resorption marker serum c-telopeptide (sCTX). Cycling of 25(OH)D was assessed by calculating the difference between the winter (nadir) and summer (peak) 25(OH)D and for ease of interpretation; all changes were expressed as positive values. Dependent variables analysed were absolute values for autumn femoral neck and lumbar spine BMD, BMC and bone area, and absolute sCTX in each season. Also, change in sCTX from summer to winter and change in the DXA bone indices from autumn to spring were analysed. ANCOVA, controlling for summer and winter 25(OH)D status, age, BMI, socio-economic status, physical activity and dietary calcium showed no statistically significant association $(P>0.05)$ between the quartile of cycling of 25(OH)D and any bone measurement in either ethnic group except in the South Asians for absolute autumn CTX $(F=5.925, P=0.01$, Fig. 1$)$ and change in FNBMC $(F=3.111, P=0.05$, see Fig. 2$)$. Also, in Asians only, absolute autumn lumbar spine BMD approached conventional levels of statistical significance $(F=2.780, P=0.07$, see Fig. 3).

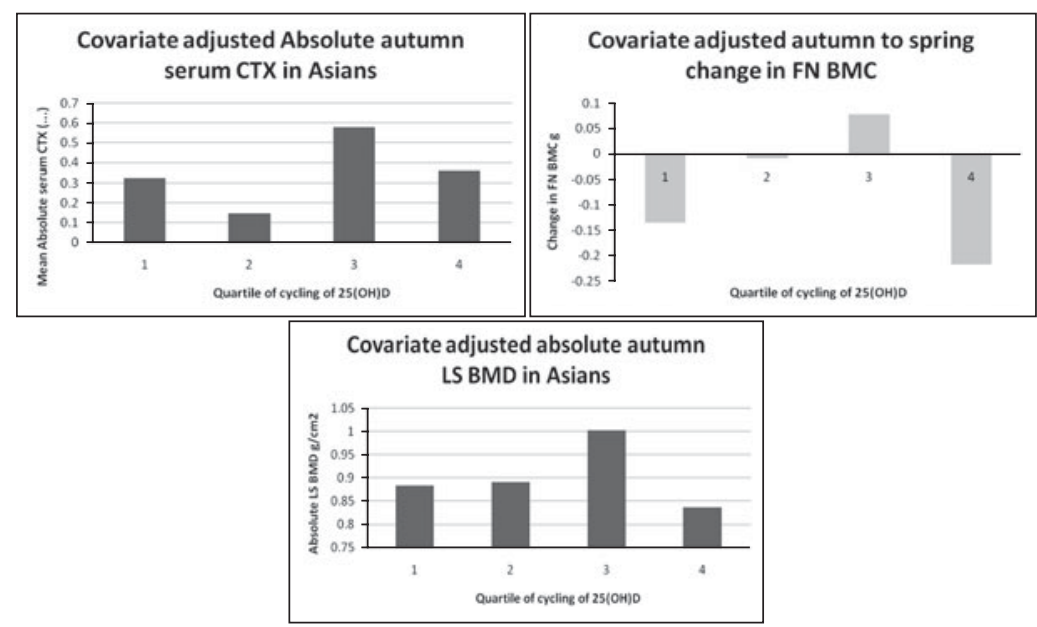

The lack of an association between cycling and most bone indices found here does not support the view that $25(\mathrm{OH}) \mathrm{D}$ cycling is detrimental to bone. However, in the South Asians only, the loss of femoral neck BMC during the year in the top and bottom quartiles but gain in the 3rd quartile, and the increased autumn sCTX in the third quartile warrants further investigation.

This study was funded by the UK Food Standards Agency (Project N05064). All views are those of the authors alone.

1. Vieth R (2004) Enzyme kinetics hypothesis to explain the U-shaped risk curve for prostate cancer vs. 25-hydroxyvitamin D in nordic countries. Int $J$ Cancer 111, 468.

2. Anderson PH \& Atkins GJ (2008) The skeleton as an intracrine organ for vitamin D metabolism. Mol Aspects Med 29, 397-406. 